\title{
ON THE EXISTENCE OF NONREGULAR ULTRAFILTERS AND THE CARDINALITY OF ULTRAPOWERS
}

BY

MENACHEM MAGIDOR

\begin{abstract}
Assuming the consistency of huge cardinals, we prove that $\omega_{3}$ can carry an ultrafilter $D$ such that $\omega_{1}^{\omega_{3}} / D$ has cardinality $\omega_{3}$. (Hence $D$ is not $\left(\omega_{3}, \omega_{1}\right)$ regular.) Similarly $\omega_{2}$ can carry an ultrafilter $D$ such that $\omega^{\omega_{2}} / D$ has cardinality $\omega_{2}$. (Hence $D$ is not $\left(\omega_{2}, \omega\right)$ regular.)
\end{abstract}

0. Introduction. When ultrafilters and ultrapowers (see [4]) were introduced, the problem of determining the cardinality of the ultrapower given the cardinality of the basis structure and of the index set became of interest (see [8]). As was already noted in [4], the problem can be completely settled, if one assumes that the ultrafilter in question is regular (see below for definition). Under the assumption of regularity the cardinality of the ultrapower has largest cardinality possible, i.e. if $M$ is a structure of cardinality $\alpha \geqslant \omega$ and $D$ is a uniform regular ultrafilter on an index set $I$ of cardinality $\beta$, then $M^{I} / D$ has cardinality $\alpha^{\beta}$.

The problem whether every ultrafilter is regular, was posed by Keisler in [9], and partial answers were obtained in the constructible universe by Jensen and then improved by Prikry [14] who showed that if $V=L$ then every uniform ultrafilter on $\omega_{n}$ is regular. Results of Benda and Ketonen [2], Kanamori [6] and finally Ketonen [10] indicate the problem is closely connected with the existence of large cardinals. In particular (see [10]) if $\kappa^{+}$ carries a uniform ultrafilter which is not $\left(\kappa^{+}, \kappa\right)$ regular, then $0^{\sharp}$ exists. On the other hand large cardinals immediately imply the existence of nonregular ultrafilters, see definition below. Note that a regular ultrafilter on $\kappa^{+}$is $\left(\kappa^{+}, \kappa\right)$ regular though the converse does not necessarily hold. If $D$ is an $\omega_{1}$ complete, nonprincipal ultrafilter on a cardinal $\kappa$, then $D$ is not regular: Note that $\kappa$ has to be at least as large as the first measurable in order to carry an $\omega_{1}$ complete ultrafilter. Keisler's problem is of course much more interesting for smaller cardinals like $\omega_{1}, \omega_{2}$ etc., and we attend this version of the problem. There is one fact known about the same problem for the continuum; if the continuum carries an $\omega_{1}$ saturated ideal then it carries a nonregular ultrafilter. Note that under this assumption the continuum is very large and this

Received by the editors August 1, 1977 and, in revised form, September 26, 1977. AMS (MOS) subject classifications (1970). Primary 02K05; Secondary 02K35.

Key words and phrases. Ultrafilter, ultraproduct, regular ultrafilter, huge cardinals. 
assumption is equiconsistent with the existence of measurable cardinals (see [16]).

Our results, which naturally assume the existence of very large cardinals, show that already $\omega_{2}$ can carry a nonregular ultrafilter and also that regularity is necessary for getting the cardinality results of [4] and [8]. In this paper we construct a model in which $\omega_{2}$ carries an ultrafilter $D$ such that for every countable structure, $M, M^{\omega_{2}} / D$ has cardinality $\omega_{2}$ which less than maximum possible (i.e. $\omega^{\omega_{2}}=2^{\omega_{2}}$ ). For $\omega_{3}$ we get even a stronger result; the ultrafilter we get on $\omega_{3}$ is not even $\left(\omega_{3}, \omega_{1}\right)$ regular and if $M$ is a structure having cardinality $\leqslant \omega_{1}$ then $M^{\omega_{3}} / D$ has cardinality at most $\omega_{3}$. Though we do not state this explicitly similar results hold for other cardinals.

The $\omega_{3}$ case also makes some progress on an old problem of Ulam. We know that if $\kappa$ is a successor cardinal $\kappa$ cannot carry a $\kappa$ complete, $\kappa$ saturated ideal, but assuming the consistency of huge cardinals, $\kappa$ can carry a $\kappa$ complete $\kappa^{+}$saturated ideal. (Most of the ideas of $\$ 2$ were derived from Kunen [11].) Naturally one wonders whether instead of relaxing the saturation requirement (i.e. the ideal is required to be $\kappa$ saturated), we can relax the completeness requirement (requiring of course that the ideal will be at least $\omega_{1}$ complete, otherwise the problem is trivial). The problem in this form was posed to us by S. Shelah. In the model we construct we can have an $\omega_{3}$ saturated, $\omega_{1}$ complete uniform ideal on $\omega_{3}$. In the same model there is a family of $\omega_{3}$ 0-1 measures defined on a field of subsets of $\omega_{3}$ such that every subset of $\omega_{3}$ is measurable with respect to at least one of the measures of the family. Compare this to 81 of [3] where the same problem is stated for $\omega_{1}$ instead of $\omega_{3}$.

1. Preliminaries. Our set theoretic notation is standard (see [5]). For terminology concerning ultrafilters and ultrapowers see [1]. Lower case greek letters usually denote ordinals. The only exception being when they denote forcing terms. $P(A)$ is the powerset of $A,|A|$ is the cardinality of $A$. If $A$ is a set of ordinals then $\bar{A}$ is the order type of $A$ and $P^{\kappa}(A)$ is the set of all subsets of $A$ having ordertype $\kappa$.

An ultrafilter $U$ on a set $I$ is uniform if for every $A \subseteq I, A \in U \rightarrow|A|=$ $|I|$. If $|I|=|J|$ then $U$ naturally induces an equivalent ultrafilter on $J$, which we shall also denote by $U$ and it should be clear from the context whether we refer to the ultrafilter on $I$ or the corresponding ultrafilter on $J$.

Let $U$ be a filter over $I, f, g$ are functions defined on $I$. We say that $f$ is equivalent to $g$ modulo $U\left(f \equiv_{U} g\right)$ if $\{i \mid i \in I, g(i)=f(i)\} \in U$. The equivalence class of $f$ with respect to $\equiv_{U}$ will be denoted by $[f]_{U}$. If $\left\langle A_{\alpha}\right| \alpha\langle\gamma\rangle$ and $\left\langle B_{\alpha}\right| \alpha\langle\lambda\rangle$ are two partitions of $I$ then $\left\langle A_{\alpha}\right| \alpha\langle\lambda\rangle$ is equivalent to $\left\langle B_{\alpha} \mid \alpha<\lambda\right\rangle$ modulo $U\left(\left\langle A_{\alpha} \mid \alpha<\lambda\right\rangle \equiv_{U}\left\langle A_{\alpha} \mid \alpha<\lambda\right\rangle\right)$ if the function $f$, defined by $f(i)=$ The only $\alpha$ such that $i \in A_{\alpha}$ is equivalent modulo $U$ to the 
function $g$ defined by $g(i)=$ The only $\alpha$ such that $i \in B_{\alpha} \cdot\left[\left\langle A_{\alpha}\right| \alpha\langle\lambda\rangle\right]_{U}$ is the equivalence class of $\left\langle A_{\alpha} \mid \alpha<\lambda\right\rangle$ with respect to $\equiv_{U}$.

If $U$ is a filter on $I$ then $A \subseteq I$ is of positive measure with respect to $U$ if $I-A \notin U$. We drop "with respect to $U$ " if the particular $U$ we refer to is evident. Note that being of positive measure with respect to an ultrafilter simply means belonging to it. $U$ is $\lambda$ complete if it is closed under intersections of length $<\lambda$ and $U$ is $\lambda$ saturated if every family of sets of positive measure with respect to $U$ such that the intersection of any two does not have positive measure with respect to $U$ has cardinality $<\lambda$.

If $I$ is a family of sets and $U$ is a filter on $I$, then $U$ is fine if for every $a \in \cup I\{i \mid a \in i\} \in U . U$ is normal if for every choice function $f$ on a set of positive measure $A$ there is a set $B \subseteq A$ of positive measure such that $f$ is constant on $B$. ( $f$ is a choice function if $f(a) \in a$ for every $a$ in its domain.) If $I$ is a family of subsets of $\lambda$, and $U I=\lambda$ then a fine filter $U$ on $I$ is called weakly normal if every choice function $f$ on $I$ is bounded with respect to $U$, i.e. there exists $\alpha<\lambda$ such that $\{i \mid f(i)<\alpha\} \in U$. Note that neither normality nor weak normality implies the other property.

An ultrafilter $U$ on $I$ is $(\alpha, \lambda)$ regular if there are $\alpha$ members of $U$ such that the intersection of any $\lambda$ of them is empty. $U$ is regular if it is $(|I|, \omega)$ regular.

$\kappa$ is huge if there is a normal, fine, $\kappa$ complete ultrafilter on $P^{\kappa}(A)$ for some $A$ such that $|A|>\kappa$. An equivalent definition (see [7]) is that there exists an elementary embedding $j$ of the universe $V$ into some transitive class $M$ such that $j(\alpha)=\alpha$ for $\alpha<\kappa, j(\kappa)>\kappa$ and $M$ contains every subset of itself having cardinality $\leqslant j(\kappa)$. Note that if $U$ is the normal, fine and $\kappa$ complete ultrafilter on $P^{\kappa}(A)$ which appears in the definition of huge cardinal, then we can get the elementary embedding of the equivalent definition by forming the ultrapower $V^{P_{k}(A)} / U$ and taking its transitive isomorph $M, j$ the canonical embedding of $V$ into $V^{P_{\kappa}(A)} / U$. In this ultrapower the identity function of $P^{\kappa}(A)$ represents the set $\{j(\alpha) \mid \alpha<j(\kappa)\}$.

The reader is well aware that many set theoretic notions have different meanings in different universes. Usually it should be clear from the context in which universe the notion applies. In case of possible doubt we use superscript on our notation to indicate the universe in which it applies, thus $P^{M}(A)$ is the powerset of $A$ in the sense of $M, \omega_{1}^{M}$ is the ordinal which $M$ considers to be $\omega_{1}$, etc.

Forcing will be very freely used in this paper, shifting between Boolean valued models and 0-1 models obtained by generic filters, as convenient. Since we shall iterate forcing constructions we use the following notation: If $B$ is a complete Boolean algebra and $T$ is a term which denotes a complete Boolean algebra in $V^{B}$ then the iterated Boolean extension $\left(V^{B}\right)^{T}$ can be considered to be one Boolean extension (see Solovay and Tennenbaum [17]). 
Let us denote the combined Boolean algebra by $B * T$. ( $B$ is of course a complete subalgebra of $B * T$.) Note that we are not distinguishing between a partially ordered set and the corresponding complete Boolean algebra. The converse procedure also applies, namely if $C$ is a complete subalgebra of $B$, $V^{C}$ can be considered to be a substructure of $V^{B}$, and $V^{B}$ is obtained from $V^{C}$ by a Boolean extension using some complete Boolean algebra of $V^{C}$. Denote a canonical term which denotes this member of $V^{C}$ by $B / C$. It is well known that $C * B / C$ is isomorphic to $B$. If $\Phi$ is a statement $[\Phi]^{B}$ is the truth value of $\Phi$ in $V^{B}$.

$\operatorname{Col}(\alpha, \beta)$ is the standard algebra which collapses every cardinal strictly between $\alpha$ and $\beta$ to $\alpha$. (See [5].) If $B$ is a complete Boolean algebra $\mathrm{Col}^{B}(\alpha, \beta)$ is a term denoting $\operatorname{Col}(\alpha, \beta)$ in the sense of $V^{B}$. As a general abuse of language we shall not distinguish between an element of the generic extension $V[G]$ ( $G$ is a $V$ generic over some partially ordered set) and a term denoting it in the appropriate forcing language. (The term of course lies in $V$.)

2. $\omega_{3}$ can carry a nonregular ultrafilter. Throughout this paper we assume the existence of a huge cardinal, so assume that $\kappa$ is a huge cardinal and assume that G.C.H. holds. (The methods of Silver [15] can be used to show that G.C.H. is consistent relative to the existence of huge cardinals.) Fix an elementary embedding $j$ of $V$ into $M$ ( $M$ a transitive class) such that $\kappa$ is the first ordinal moved by $j$ and $M^{j(\kappa)} \subseteq M$. Now we follow Kunen [11]. Exactly as in [11], one can construct a complete Boolean algebra $B$ having the following properties:

(I) $B$ has cardinality $\kappa$.

(II) $B$ satisfies the $\kappa$ chain condition.

(III) In $V^{B} \kappa$ is $\omega_{1}$.

(IV) If $C$ is a complete subalgebra of $B,|C|<\kappa$ and $\alpha<\kappa$ is a regular cardinal less than $\kappa$ then $C * \operatorname{Col}^{C}(\alpha, \kappa)$ can be completely embedded in $B$ by an embedding which is the identity on $C$. (Remember that $C$ is a subalgebra of $C * \mathrm{Col}^{C}(\alpha, \kappa)$.)

Note that in view of the fact that $M^{j(k)} \subseteq M j(B)$ is a complete Boolean algebra (not just in the sense of $M$ ) also in the sense of $V$. (Note that $j(B)$ has cardinality $j(\kappa)$ and therefore every subset of $j(B)$ lies in $M$.) $j(B)$ has the same properties (I)-(IV) where $j(\kappa)$ is substituted for $\kappa$. Note also that since $B$ satisfies the $\kappa$ chain condition $j$ is a complete embedding of $B$ into $j(B)$. (Every union of elements of $B$ can be represented as the union of less than $\kappa$ elements which is preserved by $j$, since, for $\alpha<\kappa, j(\alpha)=\alpha$.) Therefore we can assume that the domain of $B$ is $\kappa$ (hence $j$ is the identity on $B$ ) and that $B$ is a complete subalgebra of $j(B)$. Also note that $j(B)$ satisfies (even in $V$ ) the $j(\kappa)$ chain condition. 
The model we construct shall be $V^{B}$ followed by $\operatorname{Col}\left(\kappa^{+}, j(\kappa)\right.$ ) (in the sense of $\left.V^{B}\right)$. Hence we force with the Boolean algebra $D=B * \mathrm{Col}^{B}\left(\kappa^{+}, j(\kappa)\right)$. Properties (I)-(III) together with well-known facts about $\operatorname{Col}\left(\kappa^{+}, j(\kappa)\right)$ yield that the first infinite cardinals in $V^{D}$ are $\omega, \kappa, \kappa^{+}, j(\kappa)^{+}, j(\kappa)^{++}$. Hence $j(\kappa)$ is $\omega_{3}$ in the sense of $V^{D}$. Moreover since $\kappa$ and $j(\kappa)$ are inaccessible in $V$, in $V^{D}$ we have $2^{\omega}=\omega_{1}, 2^{\omega_{1}}=\omega_{2}, 2^{\omega_{2}}=\omega_{3}$, hence $\omega_{3}^{\omega_{1}}=\omega_{3}^{\omega}=\omega_{3}$.

We are now ready to define (in $V^{D}$ ) a fine filter $\mathscr{F}$ on $P^{\kappa}(j(\kappa)$ ) (which is $P^{\omega_{1}}\left(\omega_{3}\right)$ in the sense of $\left.V^{D}\right)$. This filter $\mathscr{F}$ will be the main tool for getting our results. The main fact we use about $D$ is that by (I)-(IV) applied to $j(B)$, using the fact that $B \subseteq j(B),|B|=\kappa<j(\kappa)$ there is a complete embedding of $D=B * \mathrm{Col}^{B}\left(\kappa^{+}, j(\kappa)\right)$ into $j(B)$ which is the identity on $B$. Without loss of generality we simply assume that $D \subseteq j(B)$.

In $V^{j(B)}$ we would like to define a $V^{D}$ ultrafilter on $P^{\kappa}(j(\kappa)) .\left(P^{\kappa}(j(\kappa))\right.$ is taken in the sense of $V^{D}$.) $\left(V^{D}\right.$ ultrafilter means that it is an ultrafilter in the Boolean algebra $P^{V^{D}}\left(P^{\kappa}(j(\kappa))\right)$ ). The definition we would like to use will be:

$$
A \in \mathscr{F} \leftrightarrow\{j(\alpha) \mid \alpha<j(\kappa)\} \in j(A)
$$

where $A$ is an element of $V^{D}$ which is a subset of $P^{\kappa}(j(\kappa))$. An obvious difficulty with this definition is that if $A \in V^{D} j(A)$ is an element of $M^{j(D)}$. Hence in order to decide whether $\{j(\alpha) \mid \alpha<j(\kappa)\} \in j(A)$ we have to use some generic filter over $j(D)=j(B) * \mathrm{Co}^{j(B)}\left(\kappa^{+M}, j^{2}(\kappa)\right)$. We naturally have a generic filter over $j(B)$ (in $V^{j(B)}$ ) but not over $j(D)$. Hence the question whether $A \in \mathcal{F}$ cannot be determined in $V^{j(B)}$. We overcome this difficulty (as usual in this section following Kunen [11]) by defining in $V^{j(B)}$ a sequence of elements of $j\left(\mathrm{Col}^{B}\left(\kappa^{+}, j(\kappa)\right)\right)$. Note that $j\left(\mathrm{Col}^{B}\left(\kappa^{+}, j(\kappa)\right)\right)$ is a term denoting an element of $M^{j(B)}$.

We shall consider $j\left(\mathrm{Col}^{B}\left(\kappa^{+}, j(\kappa)\right)\right)$ to be a partially ordered set such that every sequence of cardinality $\leqslant j(\kappa)$ has an upper bound, provided any two elements in the sequence are compatible (since $M^{j(\kappa)} \subseteq M$ it does not matter whether we use this closure property in $V^{j(B)}$ or in $\left.M^{j(B)}\right)$. Note that the partial order on the forcing notion is the inverse of the partial order of the corresponding Boolean algebra. The sequence (which will be denoted by $\left\langle y_{\alpha}\right| \alpha\left\langle j(\kappa)^{+}\right\rangle$) will satisfy that for every $A \in V^{D}, A \subseteq P^{j(\kappa)}(\kappa)$. The question whether $\{j(\alpha) \mid \alpha<j(\kappa)\} \in j(A)$ or not is decided over $V^{j(B)}$ by some $y_{\alpha}$. This sequence will be used to define $\mathcal{F}$.

Let $G \subseteq j(B)$ be the $V$ generic filter over $j(B)$ which generates $V^{j(B)}$ over $V$. $G \cap D$ is a $V$ generic filter over $D$ which generates $V^{D}$ over $V$. Again consider $E=\operatorname{Col}^{B}\left(\kappa^{+}, j(\kappa)\right)$ to be a forcing notion in $V^{B}$ which is closed under minimal upper bound for sequences of mutually compatible elements of length $<\kappa^{+} . K=\left\{y \mid y \in \mathrm{Col}^{B}\left(\kappa^{+}, j(\kappa)\right)\right.$; some term denoting $y$ is in $\left.G\right\}$ is a $V^{B}$ generic filter over $E$. Let $K^{1}$ be $\{j(y) \mid y \in K\}$. (What we mean by 
$j(y)$ is that we take a term denoting $y$, in the forcing language of $B, \tau$, and $j(y)$ is a realization with respect to $j(B)$ of $j(\tau)$. It is easily verified that since $j$ is the identity on $B$ it does not depend on the particular $\tau$ we picked for $y$.)

$K^{1}$ is a set of elements of $j\left(\mathrm{Col}^{B}\left(\kappa^{+}, j(\kappa)\right)\right)$ which lies in $M^{j(B)}$ since it is a subset of $M$ and has cardinality $j(\kappa)$. Any two members of it are compatible (since $y_{1}$ compatible with $y_{2}$ implies $j\left(y_{1}\right)$ compatible with $j\left(y_{2}\right)$ ), hence this set has an upper bound in $j\left(\mathrm{Col}^{B}\left(\kappa^{+}, j(\kappa)\right)\right)$ and the first member of the sequence $\left\langle y_{\alpha} \mid \alpha<j(\kappa)^{+}\right\rangle$will be picked to be such an upper bound.

Since in $V$, G.C.H. holds and $|j(B)|=j(\kappa)$ and $j(B)$ satisfies the $j(\kappa)$ chain condition, one can compute the cardinality of $P\left(P^{\kappa}(j(\kappa))\right)$ in $V^{j(B)}$ and find that it is $j(\kappa)^{+}$. Therefore let $\left\langle A_{\gamma}\right| \gamma\left\langle j(\kappa)^{+}\right\rangle$be an enumeration of $P\left(P^{\kappa}(j(\kappa))\right) \cap V^{D}$ which lies in $V^{(B)}$. Since $M^{j(\kappa)} \subseteq M$ one can easily conclude that every initial segment of $\left\langle A_{\gamma}\right| \gamma\left\langle j(\kappa)^{+}\right\rangle$lies in $M^{j(B)}$. We are now ready to define $y_{\alpha}$ by induction. $y_{0}$ was already defined. The $y_{\alpha}$ will be an increasing sequence. So for limit $\alpha$ we define $y_{\alpha}$ as an upper bound for $\left\langle y_{\beta}\right| \beta\langle\alpha\rangle$. Note that $\alpha<j(\kappa)^{+}$. We use the fact that $\left\langle y_{\beta}\right| \beta\langle\alpha\rangle$ as a sequence of cardinality $\leqslant j(\kappa)$ lies in $M^{j(B)}$. For $\alpha+1 y_{\alpha+1}$ is an extension of $y_{\alpha}$ which decides the statement $\{j(\alpha) \mid \alpha<j(\kappa)\} \in j\left(A_{\alpha}\right)$. This concludes the definition of the sequence $\left\langle y_{\alpha}\right| \alpha\left\langle j(\kappa)^{+}\right\rangle$. By construction (*) holds for our sequence where (*) is: "For every $A \in V^{D} A \subset P^{\kappa}(j(\kappa)$ ), we have for some $\alpha<j(\kappa)^{+}$that $y_{\alpha}$ decides (over $V^{j(B)}$ ) whether $\{j(\alpha) \mid \alpha<j(\kappa)\} \in j(A)$ or not". Let $\tilde{y}$ be a term denoting the sequence $\left\langle y_{\alpha}\right| \alpha\left\langle j(\kappa)^{+}\right\rangle$, which with truth value 1 satisfies the property (*).

We are ready to define in $V^{D}$ a filter on $P^{\kappa}(j(\kappa))$ as follows:

$A \in \mathscr{F}$ iff [ some member of the sequence $\tilde{y}$ forces

$$
\text { that }\{j(\alpha) \mid \alpha<j(\kappa)\} \in j(A)]^{j(B) / D}=1 .
$$

LeMma 1. $\mathscr{F}$ is a $\kappa$ complete filter $P^{\kappa}(j(\kappa))$.

The proof is similar to arguments of [11] and [12]. In particular $\mathscr{F}$ is well defined in the sense that

$$
[A=B]^{D} \leqslant[A \in \mathscr{F} \leftrightarrow B \in \mathscr{F}]^{D} .
$$

This last statement relies heavily on the particular $y_{0}$ we picked.

LEMMA 2. $\mathscr{F}$ is a fine filter on $P^{\kappa}(j(\kappa))$.

Proof. If $\alpha<j(\kappa)$ and $A=\left\{P \mid \alpha \in P, P \in P^{\kappa}(j(\kappa))\right\}$ then $A \in \mathscr{F}$ because $j(A)$ is the set $\left\{P \mid j(\alpha) \in P, P \in\left[P^{j(\kappa)}\left(j^{2}(\kappa)\right)\right]^{M^{j(D)}}\right\}$ and with truth value $1\{j(\alpha) \mid \alpha<j(\kappa)\}$ is a member of $j(A)$. Hence $\mathscr{F}$ is fine.

LEMMA 3. $\mathcal{F}$ is normal. 
Proof. Let $A$ be a set of positive measure with respect to $\mathscr{F}$ and $H$ be a choice function on $A\left(A, H \in V^{D}\right)$. Without loss of generality we can assume that it holds for $A$ and $H$ (as terms) with truth value 1 . Assume that for every $\beta<j(\kappa)\{P \mid P \in A, H(P) \neq \beta\}$ is not of positive measure, hence the set $B_{\beta}=\{P \mid P \notin A$ or $H(P) \neq \beta\} \in \mathscr{F} . j\left(B_{\beta}\right)$ is the set in $V^{j(D)}$ defined by

$$
\{P \mid P \notin j(A) \text { or } j(H)(P) \neq j(\beta)\} \text {. }
$$

By definition of $\mathscr{F}$ we know that with truth value 1 of the algebra $j(B) / D$, some condition in the sequence $\left\langle y_{\alpha} \mid \alpha<j(\kappa)^{+}\right\rangle$forces over $V^{j(B)}$

$$
\{j(\alpha) \mid \alpha<j(\kappa)\} \notin j(A) \text { or } j(H)(\{j(\alpha) \mid \alpha<j(\kappa)\}) \neq j(\beta) .
$$

Since $j(B)$ satisfies the $j(\kappa)$ chain condition we can find $\delta_{\beta}<j(\kappa)^{+}$such that with truth value 1 some condition in the sequence $\left\langle y_{\alpha} \mid \alpha<\delta_{\beta}\right\rangle$ forces (**) since $y_{\delta_{\beta}}$ is with truth value 1 an extension of each member of $\left\langle y_{\alpha} \mid \alpha<\delta_{\beta}\right\rangle$ we know that $y_{\delta_{\beta}}$ forces $(* *)$. Let $\delta=\sup _{\beta<j(\kappa)} \delta_{\beta}, \delta<j(\kappa)^{+}$. Since $y_{\delta}$ is an extension (with truth value 1) of each $y_{\delta_{\beta}}$ for $\beta<j(\kappa)$ we get that $y_{\delta}$ forces $(* *)$ for every $\beta<j(\kappa)$. We are close to getting a contradiction. We know that $A$ is of positive measure with respect to $\mathscr{F}$. Hence

$$
b=\left[\text { for some } y_{\alpha} y_{\alpha} H\{j(\alpha) \mid \alpha<j(\kappa)\} \in j(A)\right]^{j(B) / D}>0 .
$$

Without loss of generality we can assume that the $y_{\alpha}$ mentioned in the definition of $b$ is an extension of $y_{\delta}$. (Use the fact that the sequence $\left\langle y_{\alpha}\right| \alpha\left\langle j(\kappa)^{+}\right\rangle$is increasing.) Hence if we construct $\left(V^{D}\right)^{j(B) / D}$ assuming that $b$ is in the generic filter, we get in the resulting model $y_{\alpha}$ which forces

$$
\{j(\alpha) \mid \alpha<j(\kappa)\} \in j(A) \text { and } \forall \beta<j(\kappa)(j(H)(\{j(\alpha) \mid \alpha<j(\kappa)\}) \neq j(\beta)) \text {. }
$$

Hence $y_{\alpha}$ forces that $j(H)$ as an element of $M^{j(B)}$ is not a choice function on $j(A)$ because for an element of $j(A)$, namely $\{j(\alpha) \mid \alpha<j(\kappa)\}, j(H)$ on it is different from all its elements. But $H$ is, with truth value 1 , a choice function on $A$, hence $j(H)$ is, with truth value 1 , a choice function on $j(A)$. We got a contradiction and $\mathscr{F}$ is normal.

LEMMA 4. $\mathscr{F}$ is $j(\kappa)$ saturated.

Proof. $j(B)$ satisfied the $j(\kappa)$ chain condition. (Note that this implies that with truth value $1 j(B) / D$ satisfies the $j(\kappa)$ chain condition.) Let $\left\langle A_{\alpha}\right| \alpha\langle j(\kappa)\rangle$ be a sequence (in $V^{D}$ ) of sets of positive measure with respect to $\mathscr{F}$. We want to show that two members of this sequence have an intersection which is of positive measure. For every $A \subseteq P^{\kappa}(j(\kappa))$ define

$$
b(A)=[\text { some member of the sequence } y
$$

$$
\text { forces }\{j(\alpha) \mid \alpha<j(\kappa)\} \in j(A)]^{j(B) / D}
$$


(Again it does not depend on the particular term we picked to denote $A$.) It is easily verified that $b(A \cap B)=b(A) \cdot b(B) \quad b(A \cup B)=b(A)+b(B)$ $b\left(P^{\kappa}(j(\kappa))-A\right)=\overline{b(A)}$. $(A \cap B$ is some canonical term which denotes the intersections of the realizations of $A$ and $B$. Similarly for $P^{\kappa}(j(\kappa))-A$ and for $A \cup B, \overline{b(A)}$ is the boolean complement of $b(A)$.) This follows since $j(A \cap B)=j(A) \cup j(B) j(A \cup B)=j(A) \cup j(B)$ and $j\left(P^{\kappa}(j(\kappa))-A\right)=$ $j\left(P^{\kappa}(j(\kappa))\right)-j(A)$. Also note that $A$ has a positive measure with respect to $\mathscr{F}$ if and only if $b(A)>0$.

We return to the sequence $\left\langle A_{\alpha} \mid \alpha<j(\kappa)\right\rangle$. Since each of them has a positive measure $\left\langle b\left(A_{\alpha}\right)\right| \alpha\langle j(\kappa)\rangle$ is a sequence of elements of the algebra $j(B) / D$ which are all different from 0 . Hence by the fact that $j(B) / D$ satisfies the $j(\kappa)$ chain condition, we can find $\alpha, \beta<j(\kappa), \alpha \neq \beta$ such that $b\left(A_{\alpha}\right) \cap b\left(A_{\beta}\right) \neq 0$ hence $b\left(A_{\alpha} \cap A_{\beta}\right) \neq 0$ and $A_{\alpha}$ and $A_{\beta}$ has an intersection of positive measure with respect to $\mathscr{F}$, which proves that $\mathscr{F}$ is $j(\kappa)$ saturated.

LEMMA 5. $\mathcal{F}$ is weakly normal.

Proof. The facts that $\mathscr{F}$ is both normal and $j(\kappa)$-saturated imply that $\mathscr{F}$ is weakly normal, because if $F$ is a choice function on $P^{\kappa}(j(\kappa))$ we define for $\beta<j(\kappa)$

$$
A_{\beta}=\left\{P \mid P \in P^{\kappa}(j(\kappa)), F(P)=\beta\right\} .
$$

Let

$$
R=\left\{\beta \mid \beta<j(\kappa), A_{\beta} \text { has a positive measure with respect to } \mathscr{F}\right\} .
$$

Obviously if $\beta \neq \gamma A_{\beta} \cap A_{\gamma}=\varnothing$, therefore by $j(\kappa)$ saturation of $\mathscr{F},|R|<$ $j(\kappa)$. On the other hand $\cup_{\beta \in R} A_{\beta} \in \mathcal{F}$. Otherwise $B=P^{\kappa}(j(\kappa))-$ $\cup_{\beta \in R} A_{\beta}$ has a positive measure with respect to $\mathscr{F}$. Hence $F$ is constant on a subset of $B$ of positive measure. Let this constant be $\beta$, then clearly $\beta \in R$ and $A_{\beta} \cap B \neq \varnothing$ which contradicts the definition of $B$. Therefore $\cup_{\beta \in R} A_{\beta}$ $\in \mathscr{F}$. Let $\alpha=\sup R . \alpha<j(\kappa)$. Clearly

$$
\bigcup_{\beta \in R} A_{\beta} \subseteq\left\{P \mid P \in P^{\kappa}(j(\kappa)), F(P) \leqslant \alpha\right\} \in \mathscr{F}
$$

and $\mathscr{F}$ was shown to be weakly normal.

Since in $V^{D} \omega_{1}=\kappa$ and $\omega_{3}=j(\kappa)$ all the previous arguments prove:

THEOREM 1. If the existence of huge cardinal is consistent, then it is consistent that $P^{\omega_{1}}\left(\omega_{3}\right)$ carries an $\omega_{1}$ complete, normal, weakly normal and $\omega_{3}$ saturated fine filter. Besides we can assume that G.C.H. holds in the model.

Since G.C.H. implies that $\omega_{3}^{\omega_{1}}=\omega_{3}$ we can transfer $\mathscr{F}$ to an ultrafilter on $\omega_{3}$ and get 
COROllary 2. If the existence of huge cardinal is consistent then it is consistent that $\omega_{3}$ carries an $\omega_{1}$ complete $\omega_{3}$ saturated uniform filter.

This partially solves the problem of Shelah described in the introduction.

We study some further properties of $\mathscr{F}$ : Two subsets $A, B$ of $P^{\kappa}(j(\kappa))$ are equivalent modulo $\mathscr{F}\left(A \equiv_{\mathscr{F}} B\right)$ if the characteristic function of $A$ and $B$ are equivalent modulo $\mathcal{F}$ (it is equivalent to the statement that the symmetric difference between $A$ and $B, A \triangle B$, does not have positive measure with respect to $\mathscr{F})$. Assume that $b(A)=b(B)$ ( $b$ is the function defined above). Hence

$$
\begin{aligned}
b(A \triangle B) & =b\left(\left(A \cap\left(P^{\kappa}(j(\kappa))-B\right)\right) \cup\left(B \cap\left(P^{\kappa}(j(\kappa))-A\right)\right)\right) \\
& =b(A) \cdot \overline{b(B)}+b(B) \cdot \overline{b(A)}=b(A) \cdot \overline{b(A)}+b(A) \cdot \overline{b(A)}=0 .
\end{aligned}
$$

Hence $b(A)=b(B)$ implies that $A \equiv_{\mathscr{F}} B$. Since $b(A) \in j(B) / D$ and $j(B) / D$ has cardinality at most $\omega_{3}$ we get that $\equiv_{\mathscr{G}}$ has at most $\omega_{3}$ equivalence classes. More than that if $b \in j(B) / D, b \neq 0$, we can define $\mathscr{F} \uparrow b$ by $\{A \mid A \subseteq$ $\left.P^{\kappa}(j(\kappa)) ; b(A) \geqslant b\right\}$. It is easily verified that $\mathscr{F} \uparrow b$ is an $\omega_{1}$ complete normal filter on $P^{\kappa}(j(\kappa))$. Clearly if $b(A)>0 A \subseteq \mathscr{F} \uparrow b(A)$ and if $b(A)=0 P^{\kappa}(j(\kappa))$ $-A \in \mathscr{F} \uparrow b$ for every $b \in j(B) / D-\{0\}$. Each $\mathscr{F} \uparrow b$ induces naturally a 0-1 measure on a subfield of the field of all subsets of $P^{\kappa}(j(\kappa))$, namely those sets that either them or their complement is in $\mathscr{F} \uparrow b$.

Also every subset of $P^{\kappa}(j(\kappa))$ is measurable with respect to at least one of these measures. Since the cardinality of the family of these measures is at most the cardinality of $\{b \mid b \in j(B) / D\}$ which is $j(\kappa)\left(\omega_{3}^{V^{D}}\right)$ and since in $V^{D}$ $P^{\kappa}(j(\kappa))$ has cardinality $\omega_{3}$, we can assume that all these measures lie on $\omega_{3}$ and we proved

THEOREM 3. If the existence of huge cardinal is consistent then it is consistent that there is a family of $\omega_{3} 0-1 \sigma$ complete measures on $\omega_{3}$ such that every set of positive measure has cardinality $\omega_{3}$ and every subset of $\omega_{3}$ is measurable with respect to at least one of these measures.

Compare Problem 81 of [3].

We now turn to the main result of this section.

THEOREM 4. (a) Let $U$ be any ultrafilter on $P^{\omega_{1}}\left(\omega_{3}\right)$ which extends $\mathscr{F}$ then $U$ is not $\left(\omega_{3}, \omega_{1}\right)$ regular.

(b) Let $M$ be an infinite structure having cardinality $\leqslant \omega_{1}$ then $M^{P^{*}}(j(\kappa)) / U$ has cardinality $\leqslant \omega_{3}$.

From Theorem 4 we get immediately that: 
COROLlary 5. If the existence of a huge cardinal is consistent then it is consistent that $\omega_{3}$ carries a uniform ultrafilter $U$ which is not $\left(\omega_{3}, \omega_{1}\right)$ regular and such that if $M$ is a structure having cardinality $\omega \leqslant|M| \leqslant \omega_{1}$ then $M^{\omega_{3}} / U$ has cardinality at most $\omega_{3}$.

Proof of Theorem 4. In view of Keisler's [8] (b) implies (a), hence we prove (b). We first show that if $M$ is infinite and countable then $M^{P^{*}(j(\kappa))} / U$ has cardinality $\leqslant \omega_{3}$. It is easy to see that it is enough to prove it for one countable infinite structure so we show that $\langle\omega, \varepsilon\rangle^{P^{*}(j(\kappa))} / U$ has cardinality $\leqslant \omega_{3}$.

A member of $\omega^{P^{\alpha}(j(\kappa))} / U$ is of the form $[f]_{U}$ where $f$ is a function from $P^{\kappa}(j(\kappa))$ into $\omega$. (Remember that $[f]_{U}$ is the $\equiv_{U}$ equivalence class of $f$.) Our objective is to show that there are at most $\omega_{3}$ such equivalence classes. Since $\mathcal{F} \subseteq U \equiv_{\mathscr{F}}$ is a refinement of $\equiv_{U}$ and our claim will follow if we show that $\equiv_{\text {g }}$ has at most $\omega_{3}$ equivalence classes of functions from $P^{\kappa}(j(\kappa))$ into $\omega$. A function $f$ from $P^{\kappa}(j(\kappa))$ naturally induces a partition of $P^{\kappa}(j(\kappa))$ into $\omega$ classes $\left\langle A_{n}\right| n\langle\omega\rangle$ simply by defining $A_{n}=\{P \mid f(P)=n\}$. Clearly $f \equiv_{\mathscr{G}} g$ if and only if the partition corresponding to $f$ is equivalent to the partition defined by $g$. Hence instead of counting functions from $P^{\kappa}(j(\kappa))$ into $\omega$, we count $\equiv_{\mathscr{G}}$ equivalence classes of partitions of $P^{\kappa}(j(\kappa))$ into $\omega$ parts.

We need:

Lemma 6. The partitions of $P^{\kappa}(j(\kappa))\left\langle A_{n}\right| n\langle\omega\rangle$ and $\left\langle B_{n}\right| n\langle\omega\rangle$ are equivalent if and only if $A_{n} \equiv{ }^{\circ} B_{n}$ for all $n<\omega$.

Proof. If $\left\langle A_{n}\right| n\langle\omega\rangle \equiv_{\mathscr{F}}\left\langle B_{n} \mid n<\omega\right\rangle$ then for $n<\omega A_{n} \triangle B_{n}$ cannot have positive measure otherwise the functions corresponding to $\left\langle A_{n}\right| n\langle\omega\rangle$ and $\left\langle B_{n} \mid n<\omega\right\rangle$ will be different on this set of positive measure and for all $n>\omega A_{n} \equiv_{\mathscr{F}} B_{n}$.

For the converse direction assume $A_{n} \equiv B_{n}$ for all $n<\omega . C_{n}=A_{n} \Delta B_{n}$ is not of positive measure. By the $\omega_{1}$ completeness of $\mathscr{F}, C=\cup C_{n}$ is not of positive measure but clearly for $P \in P^{\kappa}(j(\kappa))-C$ the functions corresponding to $\left\langle A_{n}\right| n\langle\omega\rangle$ and $\left\langle B_{n}\right| n\langle\omega\rangle$ agree so they agree on a set in $\mathscr{F}$. $\left\langle A_{n}\right| n\langle\omega\rangle \equiv_{\mathscr{F}}\left\langle B_{n}\right| n\langle\omega\rangle$, and the lemma is proved.

Therefore an equivalence class of functions from $P^{\kappa}(j(\kappa))$ into $\omega$ is determined by an $\omega$-sequence of equivalence classes of subsets of $P^{\kappa}(j(\kappa))$ with respect to $\equiv$ F. By the remarks before Theorem 3 we have at most $\omega_{3}$ equivalence classes of subsets of $P^{\kappa}(j(\kappa))$ hence the cardinality of $\omega$ sequences of equivalence classes of subsets of $P^{\kappa}(j(\kappa))$ is at most $\omega_{3}^{\omega}=\omega_{3}$, since G.C.H. holds in our model. We have proved that there are at most $\omega_{3}$ equivalence classes of functions from $P^{\kappa}(j(\kappa))$ into $\omega$. Thus $\omega^{P^{*}(j(\kappa))} / U$ has cardinality $\leqslant \omega_{3}$.

The result we just got about ultrapowers of countable structure can be 
immediately generalized to ultraproduct of a family of countable structures and we get that this cardinality is the same as $\omega^{P^{x}}(j(\kappa)) / U$ (simply by assuming that the domain of each of the structures in the product is $\omega$ ) and we get that the ultraproduct has cardinality which is at most $\omega_{3}$.

We pass to the case where $M$ is a structure having cardinality $\omega_{1}$. Again we can assume that $M$ is $\left\langle\omega_{1}, \varepsilon\right\rangle$ and we have to show that $\left\langle\omega_{1}, \varepsilon\right\rangle^{P^{\star}(j(\kappa))} / U$ has cardinality $\leqslant \omega_{3}$. (In this case one can show that the cardinality is exactly $\omega_{3}$.) $\left\langle\omega_{1}, \varepsilon\right\rangle^{P^{k}(j(k))} / U$ is a totally ordered set. We claim that its cofinality is exactly $\omega_{3}$. We embed $\omega_{3}$ cofinally into this set by the mapping $f_{\alpha}(P)=\overline{P \cap \alpha}$ for $\alpha<\omega_{3}=j(\kappa)$. Note that since $P$ has order type $\omega_{1}$ and

$$
\{P \mid \alpha \in P\} \in \mathscr{F} \subseteq U
$$

$f_{\alpha}(P)$ is a countable ordinal for a set in $\mathscr{F}$ (hence in $U$ ). Therefore $\left[f_{\alpha}\right]_{U}$ can be considered to be an element of $\omega_{1}^{P^{p *}(j(\kappa))} / U$. We claim that the mapping $\alpha \rightarrow\left[f_{\alpha}\right]_{U}$ is cofinal in $\omega_{1}^{P^{\kappa}(j(\kappa))} / U$. Let $g$ be a function from $P^{\kappa}(j(\kappa))$ into $\omega_{1}=\kappa$. Define $h$ by $h(P)=$ the $f(P)$ th member of $P$ in the natural order of $P$. (Note that $P$ has order type $\kappa=\omega_{1}$ and $f(P)<\omega_{1}$, hence we can find the $f(P)$ th member of $P$.) $h$ is a choice function on $P^{\kappa}(j(\kappa))$, so we can use the weak normality of $\mathscr{F}$ and get $\alpha<j(\kappa)$ such that

$$
\left\{P \mid P \in P^{\kappa}(j(\kappa)), h(P)<\alpha\right\} \in \mathscr{F} .
$$

The statement implies by definition of $f_{\alpha}$ and $g$ that

$$
\left\{P \mid P \in P^{\kappa}(j(\kappa)), g(P)<f_{\alpha}(P)\right\} \in \mathscr{F} \subseteq U .
$$

Therefore $[g]_{U} \leqslant\left[f_{\alpha}\right]_{U}$ and the set $\left\{\left[f_{\alpha}\right]_{U} \mid \alpha<\omega_{3}\right\}$ is cofinal in $\omega_{1}^{P^{\alpha}(j(k))} / U$. The initial segment determined by $\left[f_{\alpha}\right]_{U}$ in $\omega_{1}^{P^{*}(j(k))} / U$ is easily seen to be isomorphic to the ultraproduct $\times_{P \in P^{*}(j(k))}\left\langle f_{\alpha}(P), \varepsilon\right\rangle / U$. On a set in $U$ the structures that appear in the product are countable, hence by the first part of the proof, the ultraproduct has cardinality $\leqslant \omega_{3}$.

Thus we proved that $\omega_{1}^{P^{*}(j(\kappa))} / U$ is a totally ordered set, having a cofinal subset of cardinality $\omega_{3}$ such that the initial segment determined by each member of this cofinal set has cardinality $\leqslant \omega_{3}$. Since the whole set is the union of these initial segments $\omega_{1}^{P^{*}(j(\kappa))} / U$ has cardinality $\leqslant \omega_{3}$ and the theorem is proved.

3. A nonregular ultrafilter on $\omega_{2}$. In this section we show how to revise the model we got in the first section to a model in which a nonregular ultrafilter lies on $\omega_{2}$ rather than $\omega_{3}$. By the proofs in $\$ 2$ we got a model in which G.C.H. holds and $P^{\omega_{1}}\left(\omega_{3}\right)$ carries a filter $\mathscr{F}$ such that:

(a) $\mathscr{F}$ is $\omega_{1}$ complete.

(b) $\mathscr{F}$ is fine, i.e. for $\alpha<\omega_{3}\{P \mid \alpha \in P\} \in \mathscr{F}$.

(c) $\mathscr{F}$ is normal; every choice function on a set of positive measure is constant on a set of positive measure. 
(d) $\mathscr{F}$ is weakly normal: every choice function on $P^{\omega_{1}}\left(\omega_{3}\right)$ is bounded below $\omega_{3}$ with respect to $\mathscr{F}$.

(e) $\mathscr{F}$ is $\omega_{3}$ saturated.

(f) There are at most $\omega_{3}$ equivalence classes modulo $\mathscr{F}$ of functions from $P^{\kappa}(j(\kappa))$ into $\omega$.

We shall not need any more properties of the model constructed in $\$ 2$ and in this section we can consider it to be the ground model $V$, in which we have the filter having properties (a)-(f).

The model which will occupy us in this section will be obtained by collapsing $\omega_{1}^{V}$ to $\omega$ by the usual Levy collapse (see [5]). Note that the cardinality of the set of forcing conditions is $\omega_{1}$. Denote the extension by $V^{1}$. In $V^{1} \omega_{1}$ is $\omega_{2}^{V}$ and $\omega_{2}$ is $\omega_{3}^{V}$. We use Theorem 2.2 in [13]. According to this theorem, if $\mathscr{F}$ is a filter on $P^{\omega_{1}}\left(\omega_{3}\right)$ which satisfies (a), (b) and (d) and we force with a set of forcing conditions having cardinality $\leqslant \omega_{1}$, in the generic extension $\mathscr{F}$ can be extended to a fine filter on $P^{\omega_{1}^{V}}\left(\omega_{3}^{V}\right)$ which is still weakly normal. Actually the proof of Theorem 2.2 in [13] yields a forcing term $\mathcal{G}$ and a mapping which assigns to every term $\tau$ an ordinal $\alpha_{\tau}<\omega_{3}^{V}$ such that every condition forces:

(a) $\mathcal{G}$ is a fine filter on $P^{\omega_{1}^{V}}\left(\omega_{3}^{V}\right)$.

(b) $\mathscr{F} \subseteq \mathcal{G}$.

(c) If $\tau$ is a choice function on $P^{\omega_{1}^{V}}\left(\omega_{3}^{V}\right)$ then $\left\{P \mid P \in P^{\omega_{1}^{V}}\left(\omega_{3}^{V}\right)<\alpha_{\tau}\right\} \in \mathcal{G}$.

THEOREM 6. In $V^{1}$ every ultrafilter $U$ which extends $\mathcal{G}$ satisfies:

(a) $U$ is not $\left(\omega_{2}, \omega\right)$ regular.

(b) If $M$ is a countable structure then $M^{P^{\omega_{1}}{ }^{\nu}\left(\omega_{3}^{\nu}\right)} / U$ has cardinality $\leqslant \omega_{2}$.

Since $\omega_{3}^{V}=\omega_{2},\left|\omega_{1}^{V}\right|=\omega$ and G.C.H. holds in $V^{1}\left|P^{\omega_{1}^{V}}\left(\omega_{2}\right)\right|=\omega_{2}$. We get:

COROLlary 7. If the existence of huge cardinal is consistent, then it is consistent to have a uniform ultrafilter $U$ on $\omega_{2}$ which is not $\left(\omega_{2}, \omega\right)$ regular and such that for every countable structure $M^{\omega_{2}} / U$ has cardinality $\leqslant \omega_{2}$.

Proof of Theorem 6. Since the set of forcing conditions used to get $V^{1}$ from $V$ has cardinality $\omega_{1}$, we can assume that it is $\omega_{1}$ endowed with the appropriate partial ordering. Similarly to the proof of Theorem 4 , if $M$ is countable the cardinality of $M^{P^{\omega_{1}}{ }_{\left(\omega_{2}\right)}} / U$ is the cardinality of $\omega^{P^{\omega_{1}}{ }^{\nu}\left(\omega_{2}\right)} / U$. So we are interested in the cardinality of $\equiv_{U}$ equivalence classes of functions from $P^{\omega_{1}^{\nu}}\left(\omega_{2}\right)$ into $\omega$. Again it will suffice to show that there are at most $\omega_{2} \equiv_{9}$ equivalence classes of such functions since $\equiv_{\mathcal{G}}$ is a refinement of $\equiv_{U}$. Actually it is rather easy to show that there are at least $\omega_{2}$ such functions by picking in $V^{1}$ a function $g$ from $\omega$ onto $\omega_{1}^{V}$, and defining for $\alpha<\omega_{3}^{V}=\omega_{2} f_{\alpha}$ : $P^{\omega_{1}^{V}}\left(\omega_{3}^{V}\right)$ by

$$
f_{\alpha}(P)=g^{-1}(\overline{P \cap \alpha}) .
$$


It is easily verified that if $\alpha \neq \beta$

$$
\left\{P \mid f_{\alpha}(P) \neq f_{\beta}(P)\right\} \in \mathscr{F} .
$$

We now prove that there are at most $\omega_{2}$ such classes of functions.

Let $\mu$ be a forcing term which is forced by every condition to be a function from $P^{\omega_{1}^{V}}\left(\omega_{3}^{V}\right)$ into $\omega$. Define a forcing term $\gamma(\mu)$ which realizes in $V^{1}$ the following function defined on $P^{\omega_{1}^{L}}\left(\omega_{2}\right)$ by:

$\gamma(\mu)(P)=$ The minimal $\beta \in \omega_{1}^{V}$ such that $\beta$ is in the generic filter which generates $V^{1}$ and $\beta \Vdash \mu(P)=n$ for some $n \in \omega$.

Let $\tau(\mu)$ be the forcing term which is realized in $V^{1}$ as the following function, defined on $P^{\omega_{1}^{V}}\left(\omega_{3}^{V}\right)$ by

$$
\tau(\mu)(P)=\text { 'The } \gamma(\mu)(P) \text { th member of } P \text { '. }
$$

Note that since $\gamma(\mu)(P)<\omega_{1}^{V}$ and $P$ has order type $\omega_{1}^{V}$ we can find the $\gamma(\mu)(P)$ th member of $P$.

$\tau(\mu)$ is a forcing term which is forced by the empty condition to be a choice function on $P^{\omega_{1}^{V}}\left(\omega_{3}^{V}\right)$. By the properties of $\mathcal{G}$ we have an ordinal $\beta(\tau)<\omega_{3}^{V}=\omega_{2}$ such that every condition forces that

$$
\{P \mid \tau(\mu)(P)<\beta(\tau)\} \in \mathcal{G} .
$$

We work in $V$. For every $\alpha \in \omega_{1}$ let $f_{\alpha}$ be a one-to-one function from $\omega$ onto $\alpha$. Define $\left\langle g_{n}^{\mu} \mid n<\omega\right\rangle$ where $g_{n}^{\mu}$ for all $n<\omega$ is a function defined on $P^{\omega_{1}}\left(\omega_{3}\right)$ by:

$$
g_{n}^{\mu}(P)=f \overline{p \cap \beta(\mu)}(n) .
$$

Note that $g_{n}^{\mu}$ depends just on $n$, and $\beta(\mu)$ and not on $\mu$. Define $\left\langle h_{n}^{\mu}\right| n\langle\omega\rangle$ as follows: $h_{n}: P^{\omega_{1}}\left(\omega_{3}\right) \rightarrow \omega$

$$
h_{n}^{\mu}(P)= \begin{cases}0 & \text { if for no } k \in \omega g_{n}^{\mu}(P) \Vdash \mu(P)=k, \\ k+1 & \text { if } g_{n}^{\mu}(P) \Vdash \mu(P)=k .\end{cases}
$$

The main reason for defining $\left\langle h_{n}^{\mu}\right| n\langle\omega\rangle$ is that $\beta(\mu)$ together with $\left\langle\left[h_{n}^{\mu}\right]_{\mathscr{f}} n\right.$ $\langle\omega\rangle$ completely determines $[\mu]_{S}$ in $V^{1}$.

LEMMA 7. Let $\mu$ and $\mu^{1}$ be forcing terms which are forced by every condition to be functions from $P^{\omega_{1}}\left(\omega_{2}\right)$ into $\omega$. Assume that $\beta(\mu)=\beta\left(\mu^{1}\right)$ and that for every $n<\omega h_{n}^{\mu} \equiv_{\mathscr{F}} h_{n}^{\mu^{1}}$ then every condition forces that $\mu \equiv_{\mathcal{G}} \mu^{1}$.

Proof of Lemma 7. Let $A_{n}=\left\{P \mid P \in P^{\omega_{1}}\left(\omega_{3}\right), h_{n}^{\mu}(P)=h_{n}^{\mu^{1}}(P)\right\}$. By assumption $A_{n} \in \mathscr{F}$ for $n<\omega$, hence $A=\bigcap_{n<\omega} A_{n} \in \mathscr{F}$ since $\mathscr{F}$ is $\omega_{1}$ complete.

In $V^{1}$ define a set $B$ by

$$
B=\left\{P \mid P \in P^{\omega_{1}^{V}}\left(\omega_{3}^{V}\right), \tau(\mu)(P)<\beta(\mu), \tau\left(\mu^{1}\right)(P)<\beta(\mu)=\beta\left(\mu^{1}\right)\right\} .
$$


We claim that every condition forces that for $P \in B \cap A \mu(P)=\mu^{1}(P)$, hence $\mu \equiv_{\mathcal{G}} \mu^{1}$. (Note that $A \in \mathscr{F} \subseteq \mathscr{F}^{\prime}$.) Assume otherwise and let $P \in$ $P^{\omega_{1}^{V}}\left(\omega_{3}^{V}\right)$ and $\delta<\omega_{1}^{V}$ such that:

$$
\delta \Vdash P \in B \wedge \mu(P) \neq \mu^{1}(P) \wedge P \in A .
$$

Since $\delta \Vdash P \in B \delta \Vdash \tau(\mu)(P)<\beta(\mu)$.

Without loss of generality we can assume that $\delta \Vdash \gamma(\mu)(P)=\rho$ for some $\rho<\omega_{1}^{V}$. By definition $\gamma(\mu)$ we know that $\rho$ and $\delta$ are compatible since $\delta$ forces that $\gamma(\mu)(P)$ is in the generic filter. Also $\rho \Vdash \mu(P)=n$ for some $n<\omega$. By definition of $\tau(\mu)$ we get that

$$
\delta \Vdash \rho=\gamma(\mu)(P)<\overline{P \cap \beta(\mu)} .
$$

Since $\rho<\overline{P \cap \beta(\mu)}, \rho=f \overline{P \cap \beta(\mu)}(m)=g_{m}^{\mu}(P)$ for some $m<\omega$. We used the fact that $f_{\alpha}$ is onto $\alpha$ for all $\alpha<\omega_{1}^{V}$. By definition of $h_{m}^{\mu}(P)$ we get that $h_{m}(P)=n+1$ (because $\rho \Vdash \mu(P)=n$ ).

Note that $P \in A$, therefore $h_{m}^{\mu}(P)=h_{m}^{\mu^{1}}(P)$. By definition of $h_{m}^{\mu^{1}}(P)$ $g_{m}^{\mu^{\prime}}(P) \Vdash \mu^{1}(P)=n$. But since $\beta(\mu)=\beta\left(\mu^{1}\right) g_{m}^{\mu^{\prime}}=g_{m}^{\mu}$ (remember that $g_{m}^{\mu}$ depends just on $\beta(\mu)$ ), and $g_{m}^{\mu^{\prime}}(P)=\rho$, therefore $\rho \Vdash \mu^{1}(P)=n=\mu(P)$ which contradicts the fact that $\delta$ is compatible with $\rho$ and that $\delta \Vdash \mu(P) \neq$ $\mu^{1}(P)$.

We resume the proof of Theorem 6, and we argue in $V$. By property (f) of $\mathscr{F}$ we have $\omega_{3}$ possible value of $\left[h_{n}^{\mu}\right]_{\mathscr{F}}$ because $h_{n}^{\mu}$ is a function from $P^{\omega_{1}}\left(\omega_{3}\right)$ into $\omega$. Since $\omega_{3}^{\omega}=\omega_{3}$ the cardinality of possible sequences of the form $\left\langle\left[h_{n}^{\mu}\right]_{\mathscr{F}} n\langle\omega\rangle\right.$ is $\omega_{3}$. The cardinality of possible pairs of the form $\left\langle\beta(\mu),\left\langle\left[h_{n}^{\mu}\right]_{\mathscr{f}} n\langle\omega\rangle\right\rangle\right.$ is again $\omega_{3}$. By Lemma 7 if for $\mu$ and $\mu^{1}$

$$
\left\langle\beta(\mu),\left\langle\left[h_{n}^{\mu}\right]_{\mathscr{F}} \mid n\langle\omega\rangle\right\rangle=\left\langle\beta\left(\mu^{1}\right),\left\langle\left[h_{n}^{\mu^{1}}\right]_{\mathscr{F}} \mid n\langle\omega\rangle\right\rangle\right.\right.
$$

then $\mu \equiv_{\mathcal{S}} \mu^{1}$, hence in $V^{1}$ we have at most $\omega_{3}^{V}$ different $\equiv_{\mathcal{Q}}$ equivalence classes of functions from $P^{\omega_{1}^{V}}\left(\omega_{3}^{V}\right)$ into $\omega$. The theorem is proved in view of the fact that in $V^{1} \omega_{2}=\omega_{3}^{V}$.

\section{REFERENCES}

1. J. L. Bell and A. B. Slomson, Models and ultraproducts. An introduction, North Holland, Amsterdam, 1969.

2. M. Benda and J. Ketonen, Regularity of ultrafilters, Israel J. Math. 17 (1974), 231-240.

3. P. Erdös and A. Hajnal, Unsolved problems in set theory, Axiomatic Set Theory, Proc. Sympos. Pure Math., vol. 13, part I, Amer. Math. Soc., Providence, R. I., 1971, pp. 17-48.

4. T. Frayne, A. C. Morel and D. Scott, Reduced direct products, Fund. Math. 51 (1962), 195-228.

5. T. J. Jech, Lectures in set theory with particular emphasis on the method of forcing, Lecture Notes in Math., vol. 217, Springer-Verlag, Berlin, and New York, 1971.

6. A. Kanamori, Weakly normal filters and irregular ultrafilters, Trans. Amer. Math. Soc. 220 (1976), 393-399.

7. A. Kanamori, W. N. Reinhardt and R. M. Solovay, Elementary embeddings and strong axioms of infinity, Ann. Math. Logic 13 (1978), 73-116. 
8. H. J. Keisler, On cardinalities of ultraproducts, Bull. Amer. Math. Soc. 70 (1964), 644-647.

9. $\quad$ A survey of ultraproducts in logic, Methodology and Philosophy of Science, Proc. of the 1964 Internat. Congress, Amsterdam, 1972, pp. 112-125.

10. J. Ketonen, Nonregular ultrafilters and large cardinals, Trans. Amer. Math. Soc. 224 (1976), 61-73.

11. K. Kunen, On saturated ideals, J. Symbolic Logic 43 (1978), 65-76.

12. K. Kunen and J. H. Paris, Boolean extensions and measurable cardinals, Ann. Math. Logic 2 (1971), 359-377.

13. M. Magidor, On the singular cardinals problem. II, Ann. of Math. 106 (1977), 514-547.

14. K. Prikry, On a problem of Gillman and Keisler, Ann. Math. Logic 2 (1970), 179-187.

15. J. Silver, G.C.H. and large cardinals (to appear).

16. R. M. Solovay, Real valued measurable cardinals, Axiomatic Set Theory, Proc. Sympos. Pure Math., vol. 13, part I, Amer. Math. Soc., Providence, R. I., 1971, pp. 397-428.

17. R. M. Solovay and S. Tennenbaum, Iterated Cohen extensions and Souslin problem, Ann. of Math. (2) 94 (1971), 201-245.

Department of Mathematics, Ben-Gurion University of the Negev, Beer-Sheva, IsRael

Current address: Institute of Mathematics, Hebrew University, Jerusalem, Israel 